\title{
The Loneliness of Long-Term Thiopurine Monotherapy for Patients with Inflammatory Bowel Disease
}

\author{
Robert N. Lopez ${ }^{1}$ Andrew S. Day ${ }^{2}$ \\ Published online: 7 August 2020 \\ (c) Springer Science+Business Media, LLC, part of Springer Nature 2020
}

The long-term management of inflammatory bowel disease (IBD) relies on the use of one or more interventions aimed at suppressing inflammation and preventing recurrence. Furthermore, with increasing emphasis upon treat-to-target concepts, there is the paramount goal of achieving and maintaining mucosal healing $(\mathrm{MH})$, which alters the disease course and reduces risks of adverse outcomes, including hospitalisation and surgery. [1, 2] While keeping these objectives in mind, any therapeutic choice must ensure an appropriate balance between efficacy and adverse effects.

Over the last 40 years, the thiopurines (TP) have been used as a mainstay in the maintenance of remission, especially in Crohn disease (CD) [3]. Azathioprine and 6-mercaptopurine can be used as monotherapy or in combination with other maintenance drugs, such as amino-salicylates or biologic therapies. Though initial assessment of thiopurine methyl transferase activity may guide initial dosing, therapeutic drug monitoring (TDM) utilising the measurement of 6-thioguanine nucleotides (6-TGN) and 6 methyl-mercaptopurine (6-MMP) is recognised as an important aspect of optimising outcomes with TP. [3] TDM enables individualised therapy, whilst potentially reducing the risks of bone marrow suppression and hepatotoxicity.

In the current issue of Digestive Diseases and Sciences, Balram and coauthors [4] describe the outcomes of TP monotherapy in a group of Canadian adults with IBD. This retrospective assessment included 100 subjects with $\mathrm{CD}$ and 48 with ulcerative colitis (UC) who were managed with a $\mathrm{TP}$ and were in clinical remission for at least 3 months. The primary outcome was the continuation of clinical remission

Andrew S. Day

andrew.day@otago.ac.nz

1 Paediatric Gastroenterology, Paeds in a Pod, Brisbane, QLD, Australia

2 Department of Paediatrics, CureKids Chair of Paediatric Research, University of Otago Christchurch, Christchurch, New Zealand during a period of 5 years. Thirty-four (23\%) of the patients remained in clinical remission on TP monotherapy after the period of observation. Fifty-five patients were classified as having a relapse despite their maintenance TP and almost all of those patients who developed adverse effects discontinued their drug.

Although standard dosage regimens were used, TDM was not available to support optimisation of dosing in this cohort. Such data may have enhanced outcomes further in the 55 subjects who had relapses by ensuring sufficient drug was administered in order to provide therapeutic 6TGN levels. Furthermore, some of the subjects who suffered adverse effects (e.g. those with elevated transaminases or leukopaenia) may have been able to avoid these if TDM was employed to adjust doses accordingly.

Furthermore, this cohort was not defined in terms of the full extent of remission upon starting a TP or subsequently. Although clinical remission for at least 3 months was an inclusion criterion, rates of biochemical remission or $\mathrm{MH}$ at baseline were not detailed, nor were rates of $\mathrm{MH}$ defined after commencement of TP therapy. TPs may induce remission (albeit with delayed onset), and are associated with modest rates of $\mathrm{MH}$. In this regard, Qiu et al. [5] recently reported their experience with TP in 130 Chinese patients with $\mathrm{CD}$. $\mathrm{MH}$ was detected in $38 \%$ after 12 months and in $46 \%$ of the cohort after 3 years of TP. Furthermore, the presence of $\mathrm{MH}$ at 12 months was associated with key outcomes at 36 months including biochemical remission and $\mathrm{MH}$.

In addition to TP pharmacodynamics, other factors, such as the time of onset, may also influence the outcomes of these drugs. The time to initiation with TP therapy in the current study [4] was, on average, 7.5-8.5 years after diagnosis of CD or UC, respectively. There is conflicting evidence on the merits of early versus later initiation of a TP in individuals with IBD [6]. Early initiation of a TP in children has been associated with reduced need for surgical intervention in some but not all reports [7]. Whilst several studies have intimated a lack of benefit with early initiation with a 
TP, a more discerning interpretation of their findings suggest that stratification of TP use on the basis of predicted disease course rather than necessarily early in the disease course was protective insofar as deriving optimal benefit balanced against potentially unnecessary use [6].

In concert with disease duration, disease phenotype may also be relevant to TP outcomes. Around one-third of the subjects with $\mathrm{CD}$ in the current report had complicated disease. Magro et al. [8] demonstrated that the initiation of a TP within 2 years of diagnosis in individuals with uncomplicated CD delayed the progression from inflammatory phenotype to stricturing or fistulising phenotypes. Disease type may also influence the response to TP: the small number of subjects in the current study precluded further subset analysis, such as the response in those with $\mathrm{CD}$ compared with those with UC.

Being able to more clearly predict those who might respond well to a TP is a further important consideration. Biomarkers such as faecal calprotectin have become increasingly important for documenting disease activity, monitoring disease and also for predicting therapeutic outcomes. Serum CRP levels show some promise as a predictor of response to TP therapy, with persistently raised levels suggesting consequent treatment failure [9]. Establishing key biomarkers at baseline, or within the initial period of TP dosing, would help predict those individuals who might respond better.

In summary, this important report highlights several key aspects of TP monotherapy in individuals with IBD, with a low rate of long-term utility evident. Rather than taking the glass half-empty approach to these data, optimisation of TPs may enhance outcomes. TDM is important to individualise dosing, optimising efficacy and avoiding adverse outcomes. Establishment of the optimal timing of onset of TP along with predictive markers and indicators of response (including $\mathrm{MH}$ ) are also essential.

\section{Compliance with Ethical Standards}

Disclosures No conflict of interest.

\section{References}

1. Peyrin-Biroulet L, Sandborn W, Sands BE, et al. Selecting therapeutic targets in inflammatory bowel disease (STRIDE): determining therapeutic goals for treat-to-target. Am J Gastroenterol. 2015;110:1324-1338.

2. Shah SC, Colombel JF, Sands BE, Narula N. Systematic review with meta-analysis: mucosal healing is associated with improved long-term outcomes in Crohn's disease. Aliment Pharmacol Ther. 2016;43:317-333.

3. de Boer NKH, Peyrin-Biroulet L, Jharap B, et al. Thiopurines in inflammatory bowel disease: new findings and perspectives. $J$ Crohns Colitis. 2018;12:610-620.

4. Balram N, Lubov J, Theoret $\mathrm{Y}$ et al. Poor drug sustainability in inflammatory bowel disease patients in clinical remission on thiopurine monotherapy. Dig Dis Sci. (Epub ahead of print). https:// doi.org/10.1007/s10620-020-06427-8.

5. Qiu Y, Chen BL, Mao R, et al. Endoscopy assessment at 1-year identifies long-term responders to thiopurines maintenance therapy in patients with crohn's disease. Medicine (Baltimore). 2015;94:e1204.

6. Mantzaris GJ. Thiopurines and methotrexate use in IBD patients in a biologic era. Curr Treat Options Gastroenterol. 2017;15:84-104.

7. Chhaya V, Pollok RCG, Cecil E, et al. Impact of early thiopurines on surgery in 2770 children and young people diagnosed with inflammatory bowel disease: a national population-based study. Aliment Pharmacol Ther. 2015;42:990-999.

8. Magro F, Rodrigues-Pinto E, Coelho R, et al. Is it possible to change phenotype progression in Crohn's disease in the era of immunomodulators? Predictive factors of phenotype progression. Am J Gastroenterol. 2014;109:1026-1036.

9. Cornish JS, Wirthgen E, Dabritz J. Biomarkers predictive of response to thiopurine therapy in inflammatory bowel disease. Front Med (Lausanne). 2020;7:8.

Publisher's Note Springer Nature remains neutral with regard to jurisdictional claims in published maps and institutional affiliations. 